\title{
Effect of Plasma Volume Loss during Graded Exercise Testing on Blood Lactate Concentration
}

\author{
James A. DAVIS, Ralph ROZENEK, Derek M. DECICCO, Michael T. CARIZZI, and \\ Patrick H. PHAM \\ Laboratory of Applied Physiology, Department of Kinesiology, California State University/Long Beach, Long Beach, CA, USA
}

\begin{abstract}
Previous studies have shown that plasma volume (PV) loss can be a confounding variable in the interpretation of changes in blood constituents. We examined the effect of PV loss on three features of the blood lactate versus work-rate relationship, namely, slight blood lactate increase during the early stages of graded exercise testing (GXT); work rate at the onset of a systematic increase in blood lactate, i.e., lactate threshold (LT); and work rate at a blood lactate concentration of $4 \mathrm{~mm}$, i.e., onset of blood lactate accumulation (OBLA). Fourteen subjects underwent cycle ergometer GXT. Blood samples were obtained at rest and at the end of each 3-min work-rate increment and analyzed for hematocrit and lactate concentration. For exercise levels up to and including LT, PV loss was relatively stable at ap-
\end{abstract}

proximately $2.8 \%$. Beyond LT, PV loss accelerated. From the first work rate to $L T$, blood lactate concentration uncorrected for PV loss increased $0.24 \pm 0.07 \mathrm{mM}(P<0.05)$. After correction for PV loss, the increase was $0.21 \pm 0.08 \mathrm{mM}(P<0.05)$. These mean increases were not significantly different from each other. For the four exercise levels above LT common to most subjects, PV-corrected lactate values were significantly lower than uncorrected values. Correction of lactate values for PV loss did not alter LT for any subject, but it did result in a significant increase in OBLA. Thus, PV loss has the potential to be a confounding variable for the interpretation of blood lactate parameters that are determined at exercise levels above LT.

Key words: cycle ergometer, graded exercise testing, hemoconcentration, lactate threshold, onset of blood lactate accumulation.

$\mathrm{D}$ uring graded exercise testing (GXT), blood lactate concentration has a characteristic response pattern. At low and moderate exercise levels, it demonstrates a small increase [1]. At higher exercise levels, a marked, curvilinear increase is evident [1]. The exercise level at the beginning of the curvilinear increase in blood lactate concentration is called the lactate threshold [2]. Thus, the lactate threshold (LT) represents the onset of a metabolic acidosis [2]. The LT is a valuable measurement in clinical exercise testing settings for patients with heart and/or lung disease [3] and is well correlated to endurance performance [6]. The onset of blood lactate accumulation (OBLA) is defined as the work rate at which blood lactate concentration reaches $4 \mathrm{mM}$ during GXT [4]. This measurement can be used for the assessment of cardiorespiratory health and the evaluation of exercise training programs [5], and, like the LT, it is well correlated to endurance performance [6]. With the use of both LT and OBLA, exercise intensity can be partitioned into mild (below LT), moderate (from LT to OBLA), and intense (above OBLA) domains [5].

Two explanations have been suggested in the literature for the small increase of blood lactate concentration during the early work rates of GXT $[11,12]$. One is that these work rates engender a small amount of anaerobic metabolism that result in a small increase in blood lactate concentration [11]. A second explanation is that some lactate is produced by the forearm muscles during the early stages of cycle ergometer GXT as the subject attempts to stabilize the upper torso during that mode of exercise [12]. There is a third explanation that to our knowledge has not been considered. It may be that some plasma is lost from the vascular space during the early stages of GXT, thereby concentrating the constituents in the vascular space including lactate.

The characteristic blood lactate pattern of response described above is for blood lactate that has not been corrected for exercise-induced plasma volume (PV) loss. Exercise causes a reduction of $\mathrm{PV}$ as fluid moves from the vascular space into both the interstitial and intracellular spaces of contracting muscle [7]. Two mechanisms have been suggested to explain the efflux of fluid from the vascular space during exercise. One is that increases in arterial blood pressure and muscle contraction on venules result in an increased capillary hydrostatic pressure that pushes plasma fluid into the extravascular space [8]. Another is that the metabolic waste products resulting from muscle

Received on Dec 24, 2006; accepted on Feb 21, 2007; released online on Feb 24, 2007; doi:10.2170/physiolsci.RP014406 Correspondence should be addressed to: James A. Davis, Laboratory of Applied Physiology, Department of Kinesiology, California State University, Long Beach, Long Beach, CA 90840-4901, USA. E-mail: jad@csulb.edu 
contraction (e.g., lactate, potassium, and phosphate) increase the osmolality of the interstitial space, creating an osmotic force that pulls plasma fluid from the vascular space into the extravascular space [9]. The result of the exercise-induced PV decrease is to concentrate the constituents of the vascular space, thus causing a hemoconcentration [10].

Previous studies have shown that PV loss can be a confounding variable in the interpretation of changes in blood constituents with exercise [13-15]. Because the loss of PV during GXT has the potential to alter the blood lactate versus work-rate relationship, the present study was designed to examine three features of that relationship: (a) the small increase in blood lactate concentration during the early portion of the GXT, (b) the work rate at the onset of a systematic increase in blood lactate concentration (i.e., LT), and (c) the work rate at a blood lactate concentration of $4 \mathrm{mM}$ (i.e., OBLA).

\section{METHODS}

Fourteen subjects ( 8 males and 6 females) were recruited from the California State University, Long Beach campus and surrounding communities. The Physical Activity Readiness Questionnaire (PAR-Q) health-screening questionnaire [16] was administered to each person who expressed an interest in the study. The activity level of the subjects ranged from sedentary (not engaged in a formal exercise training program) to very active (competing in endurance sports). The mean \pm standard error (SE) for age, height, and body mass for the 14 subjects were $29.7 \pm$ $1.9 \mathrm{yr}, 170.7 \pm 2.7 \mathrm{~cm}$, and $69.7 \pm 4.9 \mathrm{~kg}$. Standard procedures were used to measure height and body mass. Before the study began, the subjects read and signed a consent form approved by the Institutional Review Board for the Protection of Human Subjects of California State University, Long Beach.

Exercise testing was conducted on a Monark cycle ergometer (Quinton Instruments, Seattle, WA, USA). The GXT protocol consisted of work rate increases every 3 min to the limit of tolerance. The size of the work-rate increment varied among subjects. For sedentary subjects, it was typically $15 \mathrm{~W}$. For very active subjects, it was typically $35 \mathrm{~W}$. The rationale for the two protocols was based on our previous experience that a protocol that is too fast for sedentary subjects (e.g., $35 \mathrm{~W}$ per $\mathrm{min}$ ) results in a test that is too short for data collection purposes, and one that is too slow for the very active subjects (e.g., $15 \mathrm{~W}$ per $\mathrm{min}$ ) results in a test that is unduly long. The subjects were instructed to rest their hands on the cycle ergometer handlebars throughout all but the maximal portion of the GXT. This was done to minimize the production of lactate by the forearm muscles as a result of gripping the handlebars. After sitting on the cycle ergometer for $20 \mathrm{~min}$ to eliminate the potential influence of posture change on the measurement of blood constituents [17], a resting blood sample was taken from the subjects. Graded exercise testing then commenced with blood sampling during the last $30 \mathrm{~s}$ of each work-rate increment. Capillary blood was collected from a free-flowing digit puncture. The subject's hand was placed in a heating pad throughout the GXT except at the time of blood collection. Each blood sample was analyzed for hematocrit and lactate. Hematocrit was determined in duplicate by the microhematocrit method following the procedures of Convertino et al. [18]. Hematocrit readings were corrected for trapped plasma and whole-body hematocrit with the factors of 0.96 and 0.91 , respectively $[18,19]$. Percent change in PV or delta PV $(\triangle \mathrm{PV})$ from rest to each exercise level was calculated using the corrected hematocrit values according to the equation of van Beaumont [20]. Lactate concentration was determined in duplicate using an automated lactate analyzer (YSI 2300, Yellow Springs Instruments, Yellow Springs, Ohio, USA). The analyzer was calibrated before and during each set of samples according to manufacturer recommendations. To correct the blood lactate concentration values for $\triangle \mathrm{PV}$, the blood lactate concentration values were multiplied by $[1+(\Delta \mathrm{PV} / 100])$. From the blood lactate concentration versus work-rate plot for each subject, the LT was determined as the work rate at which the lowest lactate value was greater than $0.5 \mathrm{mM}$ lower than the following one [21]. From that same plot, OBLA was determined as the work rate at a blood lactate concentration of $4 \mathrm{mM}$ using the method of Bishop et al. [22]. Heart rate was determined each minute from standard electrocardiogram leads. Predicted maximal heart rate (HR) was calculated as 220 - age.

All statistical analyses were performed using the Statistical Package for the Social Sciences 14.0 for Windows. The alpha level chosen for statistical significance was $P<$ 0.05 . The results are presented throughout as the mean \pm $\mathrm{SE}$. The unpaired $t$-test was used to compare the $\Delta \mathrm{PV}$ responses and blood lactate concentration responses between the male and female subjects. No differences were found, so the statistical analysis was performed on the entire sample of subjects $(n=14)$. The one-sample $t$-test was used to test the hypothesis of no change in $\triangle \mathrm{PV}$ from rest to the LT and from rest to maximal exercise. The paired $t$ test was employed to compare PV from rest to the LT and from rest to maximal exercise. The one-sample $t$-test was also used to test the hypothesis of no change in uncorrected and PV-corrected blood lactate concentration from the start of the GXT to the LT. The paired $t$-test was employed to compare the mean increases of uncorrected and PV-corrected blood lactate concentration from the start of the GXT to the LT. Regarding the relationship of blood lactate concentration and exercise level, a repeated-measures two-factor analysis of variance (ANOVA) was employed to determine if a significant $F$ ratio was found for one or both factors (exercise level and blood lactate concentra- 
tion that was uncorrected and corrected for $\triangle \mathrm{PV}$ ) and the interaction term. If the interaction term was significant, a paired $t$-test (with Bonferroni correction of the alpha level) would be used to compare the mean values at each exercise level for blood lactate concentration uncorrected and corrected for $\triangle \mathrm{PV}$. To compare the mean values for $\mathrm{LT}$ and for OBLA determined from blood that was uncorrected and corrected for $\Delta \mathrm{PV}$, the paired $t$-test was used.

\section{RESULTS}

The exercise levels that were common to nearly all of the subjects were the two before the LT and four after it. One subject's GXT ended at two exercise levels after the LT. The GXT for two subjects ended at three exercise levels after the LT. Figure 1 displays the relationship of $\triangle \mathrm{PV}$ versus exercise level with mean \pm SE values shown at each level. For the two levels immediately prior to the LT plus the exercise level at the $\mathrm{LT}, \triangle \mathrm{PV}$ is relatively stable at about $-2.8 \%$. Beyond the LT, PV decreased in an approximately linear manner, falling about $3.2 \%$ per exercise level.

From the first work rate to the LT, the blood lactate concentration uncorrected for PV changes had an increase of $0.24 \pm 0.07 \mathrm{mM}$. After correction for the PV changes, the increase was $0.21 \pm 0.08 \mathrm{mM}$. The mean increases, before and after correction for PV changes, were not significantly different from each other, but they were significantly different from no change in blood lactate concentration from the first work rate to the LT.

Shown in Fig. 2 is the relationship between blood lactate concentration and exercise level for blood lactate concentration values that are uncorrected for $\Delta \mathrm{PV}$ and that are corrected for $\triangle \mathrm{PV}$. The mean differences between uncorrected and PV-corrected blood lactate concentration were not significantly different for the first two exercise levels or the exercise level at the LT, but they were significantly different at each of the other exercise levels (Ex), i.e., Ex4, Ex-5, Ex-6, and Ex-7 of Fig. 2. For each exercise level beyond the LT, all subjects had lower PV-corrected blood lactate values except for two subjects at Ex-4 and one at Ex-5. For these three exceptions, the PV-corrected blood lactate values were just slightly higher by 0.04 and 0.07 $\mathrm{mM}$ for the two subjects at Ex-4 and $0.10 \mathrm{mM}$ for the one subject at Ex-5.

All subjects had the same LT value determined from uncorrected and PV-corrected blood lactate, therefore, the mean values of $104.0 \mathrm{~W}$ (with $\mathrm{SE}$ of $\pm 11.6 \mathrm{~W}$ ) were not significantly different. The mean \pm SE values for OBLA determined from uncorrected and PV-corrected blood lactate were $149.7 \pm 15.8$ and $157.5 \pm 16.1 \mathrm{~W}$, respectively. The $7.8 \mathrm{~W}$ mean difference between the two OBLA mean values was statistically significant.

The maximal HR for the 14 subjects was $185.2 \pm 2.1$ $\mathrm{b} \cdot \mathrm{min}^{-1}$. That heart rate represents $97.3 \pm 1.2 \%$ of the age-

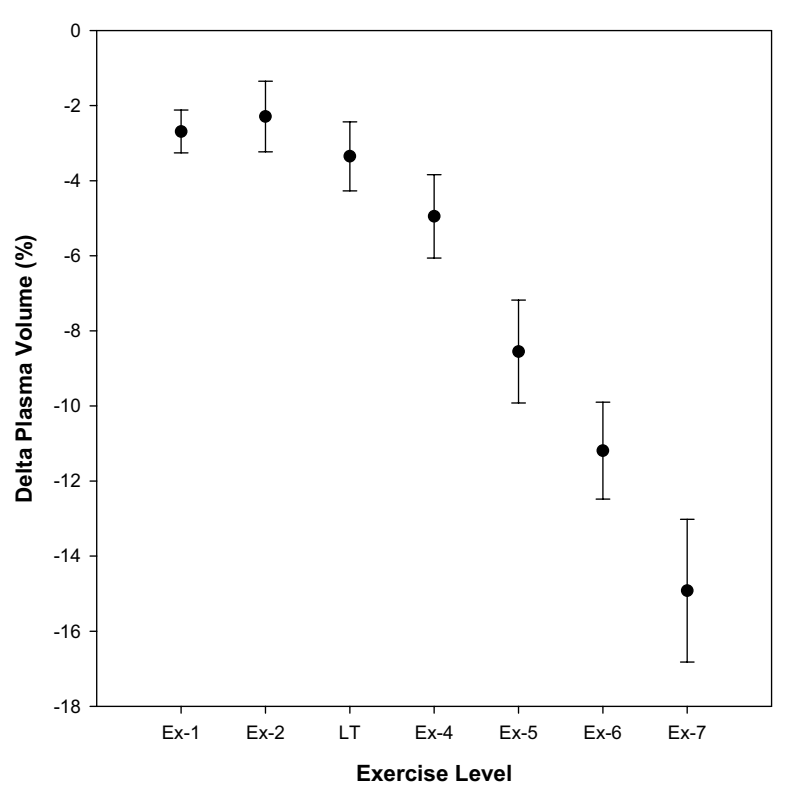

Fig. 1. The relationship between delta plasma volume and exercise level (Ex) for the 14 subjects. Shown are the mean values with standard error bars at two exercise levels before the lactate threshold (LT) and four exercise levels after the LT.

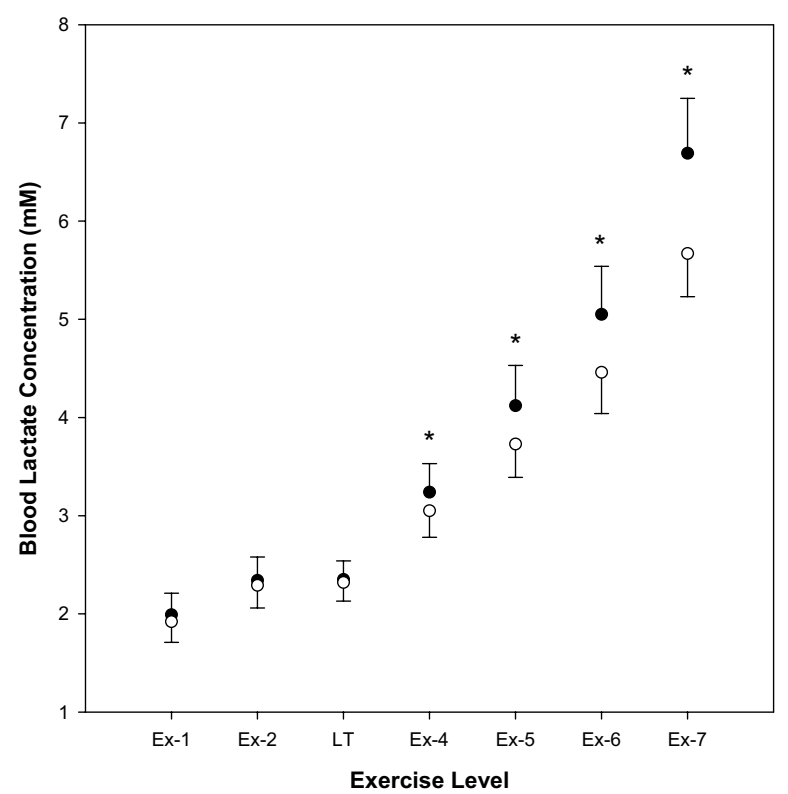

Fig. 2. The relationship between blood lactate concentration and exercise level (Ex) for the blood lactate concentration values that are uncorrected for plasma volume loss (closed circles) and that are corrected for plasma volume loss (open circles) in 14 subjects. Shown are the mean values with onesided standard error bars at two exercise levels before the lactate threshold (LT) and four exercise levels after the LT. An asterisk indicates a significant difference $(P<0.05)$ between the two mean blood lactate concentration values at a given exercise level.

predicted HR max. From rest to the LT, the decrease in PV was $3.4 \pm 0.9 \%$. From rest to maximal exercise, the decrease was $14.8 \pm 1.9 \%$. Both of these PV decreases were 
significantly different from no change in PV, and they were significantly different from each other.

\section{DISCUSSION}

Plasma volume changed very little in the early stages of the GXT for the subjects of the present study, as shown in Fig. 1. Consequently, the effect that $\triangle \mathrm{PV}$ had on two features of the blood lactate concentration versus exercise level relationship investigated in this study was minimal. A small but statistically significant increase in blood lactate concentration during the early stages of the GXT was found for the subjects of this study. However, correction of the blood lactate for $\Delta \mathrm{PV}$ did not significantly alter this increase. Thus, the results of this study do not support the possibility that the small increase in blood lactate concentration observed during the early portion of the GXT is due to a loss of PV. Further, correction of the blood lactate for PV loss did not alter the LT for any of the subjects in this study because the loss of PV was minimal at all work rates prior to and including the LT.

However, as exercise intensity increased beyond the LT, the loss of PV accelerated and caused the PV-corrected blood lactate mean values for each exercise level beyond the $\mathrm{LT}$ to be lower $(P<0.05)$ than the corresponding PV-uncorrected mean values (Fig. 2). This resulted in OBLA being significantly lower when determined from blood lactate values that were corrected for PV loss. The implication of this result is that PV loss during exercise testing is a confounding variable that can alter lactate measurements made at exercise intensities beyond the LT. For example, Heck et al. [23] reported that OBLA is protocol dependent. However, is it OBLA that is protocol dependent, or do different exercise testing protocols change the pattern of PV loss, giving the impression that OBLA is protocol dependent? The potential confounding influence of PV loss can be eliminated by making the lactate measurements of interest (e.g., OBLA) on PV-corrected blood. Indeed, in the recent study by Baker et al. [24], the entire analysis of the effect of two different protocols on lactate production resulting from high-intensity exercise was made on PV-corrected blood. In that study, the possibility that the difference found between the two protocols could have been in part or in full a results of differences in PV loss between the two protocols was eliminated by using PV-corrected blood for the analysis.

Convertino et al. [18], Miles et al. [25], Nagashima et al. [26], and Wilkerson et al. [27] investigated the response pattern of $\triangle \mathrm{PV}$ during GXT. Three of the studies $[18,26,27]$ found a biphasic response pattern with a relatively small decrease during the early stages of GXT followed by a steeper decrease after approximately $50 \%$ of maximal work rate. In a typical subject, the LT occurs at approximately $50 \%$ of maximal work rate [2]. The response pattern of $\Delta \mathrm{PV}$ for the subjects of the present study was similar to that found in the three studies [18, 26, 27] discussed above. On the other hand, Miles et al. [25] found that $\triangle \mathrm{PV}$ decreased in an approximately linear manner as exercise intensity increased. However, that finding was based on data for only four exercise levels with only one of those levels below $50 \%$ of maximal work rate. The $\Delta \mathrm{PV}$ for the first exercise level (at $31 \%$ of maximal oxygen uptake) in the study of Miles et al. was similar to what was found in the present study for the exercise levels below the LT. Thus, it is possible that had Miles et al. made more measurements at low and moderate work rates, they would have found a biphasic $\Delta \mathrm{PV}$ response pattern.

From rest to maximal exercise, PV demonstrated a large, significant mean decrease for the subjects of this study (see Fig. 1). The magnitude of this decrease is similar to the mean decreases previously found by van Beaumont et al. [7], Freund et al. [28], and Fry et al. [29].

Based on the results of this study, we conclude that the response pattern of blood lactate concentration during the early stages of GXT and at the LT is not altered by PV loss because PV loss was minimal during this portion of the GXT. However, because PV loss accelerated for work rates beyond the LT, OBLA was significantly lower when determined from blood lactate corrected for PV loss. Based on this latter result, we recommend that any lactate parameter lying above the LT be measured with blood that is corrected for PV loss. If that is done, the interpretation of the result can then be made without the confounding influence of PV loss on blood lactate concentration.

The authors thank David C. Bradley, PhD, and Stafford G. Cox, PhD, of the Academic Computing Services at California State University/Long Beach for statistical assistance. This research was supported by a grant from the Scholarly and Creative Activity Committee of California State University/ Long Beach, CA, USA.

\section{REFERENCES}

1. Beaver WL, Wasserman K, Whipp BJ. Improved detection of lactate threshold during exercise using a log-log transformation. J Appl Physiol. 1985;59:1936-40.

2. Davis JA. Anaerobic threshold: review of the concept and directions for future research. Med Sci Sports Exerc. 1985;17:6-18.

3. Wasserman K, Hansen JE, Sue DY, Stringer WW, Whipp BJ. Principles of Exercise Testing and Interpretation, 4th edn. Phil., PA: Lippincott, Williams, \& Wilkins; 2005, p. 86-90.

4. Sjodin B, Karlsson J. Onset of blood lactate accumulation and marathon running performance. Int J Sports Med. 1981;2:23-26.

5. Sevdahl K, Macintosh BR. Anaerobic threshold: the concept and methods of measurement. Can J Appl Physiol. 2003: 28: 299-323.

6. Bourdon P. Blood lactate transition thresholds: concepts and controversies. In: Gore CJ, editor. Physiological Tests for Elite Athletes. Champaign, IL: Human Kinetics; 2000, p. 50-65.

7. van Beaumont W, Strand JC, Petrofsky JS, Hipskind SG, Greenleaf JE. Changes in total plasma content of electrolytes and proteins with maximal exercise. J Appl Physiol. 1973;34:102-6.

8. Lundvall J, Mellander S, Westling $\mathrm{H}$, White T. Fluid transfer between blood and tissues during exercise. Acta Physiol Scand. 1972;85:258-69.

9. van Beaumont W, Undrekofler S, van Beaumont S. Erythrocyte volume, plasma volume and acid base changes in exercise and heat dehydration. J Appl Physiol. 1981:50:1255-62.

10. Kargotich S, Goodman C, Keast D, Morton AR. The influence of exercise- 


\section{PV Loss during GXT and Blood Lactate Concentration}

induced plasma volume changes on the interpretation of biochemical parameters used for monitoring exercise, training, and sport. Sports Med. 1998:26:101-17.

11. Hughson RL, Weisiger KH, Swanson GD. Blood lactate concentration increases as a continuous function in progressive exercise. J Appl Physiol. 1987;62:197581.

12. Davis JA, Caiozzo VJ, Lamarra N, Ellis JF, Vandagriff R, Prietto CA, MacMaster WC. Does the gas exchange anaerobic threshold occur at a fixed blood lactate concentration of 2 or $4 \mathrm{mM}$ ? Int J Sports Med. 1983;4:89-93.

13. Goodman C, Rogers GG, Vermaak H, Goodman MR. Biochemical responses during recovery from maximal and submaximal swimming exercise. Eur J Appl Physiol. 1985;54:436-41.

14. Gordon PM, Goss FL, Visich PS, Warty V, Denys BJ, Metz KF, Robertson RJ. The acute effects of exercise intensity on HDL-C metabolism. Med Sci Sports Exerc. 1994;26:671-677.

15. Kargotich S, Goodman C, Keast D, Fry RW, Garcia-Webb P, Crawford PM, Morton AR. Influence of exercise-induced plasma volume changes on the interpretation of biochemical data following high-intensity exercise. Clin J Sport Med. 1997;7:185-91.

16. American College of Sports Medicine. ACSM's Guidelines for Exercise Testing and Prescription, 6th edn. Phil., PA: Lippincott, Williams, \& Wilkins; 2000, p. 23.

17. Tan TH, Wilmshurst EG, Gleason RE, Soeldner JS. Effect of posture on serum lipids. N Engl J Med. 1973;289:416-19.

18. Convertino VA, Keil LC, Bernauer EM, Greenleaf JE. Plasma volume, osmolality, vasopressin, and renin activity during graded exercise in man. J Appl Physiol. 1981;50:123-8.

19. El-Sayed MS, Davies B, Morgan DB. Vasopressin and plasma volume response to submaximal and maximal exercise in man. J Sports Med Phys Fitness.
1990;30:420-25.

20. van Beaumont W. Evaluation of hemoconcentration from hematocrit measurements. J Appl Physiol. 1972;32:712-13.

21. Grassi B, Quaresima V, Marconi C, Ferrari M, Cerretelli P. Blood lactate accumulation and muscle deoxygenation during incremental exercise. J Appl Physiol. 1999;87:348-55.

22. Bishop D, Jenkins DG, Mackinnon LT. The relationship between plasma lactate parameters, $W_{\text {peak }}$ and 1-h cycling performance in women. Med Sci Sports Exerc. 1998;30:1270-5.

23. Heck H, Mader A, Hess G, Mucke S, Muller R, Hollmann W. Justification of the 4mmol/l lactate threshold. Int J Sports Med. 1985;6:117-30.

24. Baker J, Brown E, Hill G, Phillips, G, Williams R, Davies B. Handgrip contribution to lactate production and leg power during high-intensity exercise. Med Sci Sports Exerc. 2002;34:1037-40.

25. Miles DS, Sawka MN, Glaser RM, Petrofsky JS. Plasma volume shifts during progressive arm and leg exercise. J Appl Physiol. 1983;54:491-95.

26. Nagashima K, Nose H, Yoshida T, Kawabata T, Oda Y, Yorimoto A, Uemura O, Morimoto T. Relationship between atrial natriuretic peptide and plasma volume during graded exercise with water immersion. J Appl Physiol. 1995;78:217-24.

27. Wilkerson JE, Gutin B, Horvath SM. Exercise-induced changes in blood, red cell, and plasma volumes in man. Med Sci Sports. 1977;9:155-8.

28. Freund BJ, Claybaugh JR, Dice MS, Hashiro GM. Hormonal and vascular fluid responses to maximal exercise in trained and untrained males. J Appl Physiol. 1987;63:669-75.

29. Fry RW, Morton AR, Crawford GPM, Keast D. Cell numbers and in vitro responses of leucocytes and lymphocyte subpopulations following maximal exercise and interval training sessions of different intensities. Eur J Appl Physiol. 1992;64:218-27. 